\title{
GROUPS WITH PRESCRIBED AUTOMORPHISM GROUP
}

\author{
by DEREK J. S. ROBINSON*
}

(Received 10th December 1980)

\section{Introduction}

We are concerned here with question: to what extent can the structure of a group $G$ be recaptured from information about the structure of its group of automorphisms Aut $G$ ? For example, one might try to find all groups which have some specific group as their (full) automorphism group, a point of view adopted by Iyer in a recent paper [5]. Nothing is known about this question in general except the result of Nagrebeckii [7] that there are only finitely many finite groups with a given group as automorphism group.

Less ambitiously one can simply enquire whether a given group $G$ is isomorphic with the full automorphism group of at least one group. If the answer is positive, we shall say that $G$ is an automorphism group. Several classes of non-automorphism groups are known, for instance all nilpotent torsion groups of infinite exponent and all infinite Cernikov groups [10]. Further examples will be given below.

Our main result, Theorem 1, describes the structure of a group $G$ such that Aut $G$ is finite and Aut $_{c} G$, the subgroup of central automorphisms, is semisimple. An explicit construction for such groups is given, the principal constituent being a certain class of central extensions which are akin to stem extensions in the sense that the torsionsubgroup of the centre is contained in the derived subgroup.

A group $G$ with the above properties will usually be infinite, and may involve torsionfree abelian groups of complex type. However, it is not hard to see that if $H=$ Aut $G$ is finite and all its composition factors are non-abelian, then $G$ is finite. Moreover, on the basis of Theorem 1 the possibilities for $G$ may be described in terms of the internal structure of $H$. All that is required is a sufficient knowledge of the outer automorphism groups and the Schur multiplicators of the normal subgroups of $H$.

These results can be applied with advantage to finite (non-abelian) simple groups; they yield necessary and sufficient conditions for such groups to be automorphism groups (Theorem 4). It turns out that with a single exception the known finite simple groups which are automorphism groups are either complete or of the type $\operatorname{PSL}(n, 2)$. The exception is the Suzuki group $S=S z(8)$ of order 29,120 , which is the automorphism group of a proper covering group of order 58,240, and in fact of no other group.

Also discussed are the non-trivial covering groups of the known finite simple groups. It turns out that these are never automorphism groups (Theorem 5).

*Support from the National Science Foundation under grant MCS 78-02779 is gratefully acknowledged. 
Finally Theorem 1 is used to determine all the groups whose automorphism group is a finite symmetric or alternating group (Theorems 6 and 7). The finite groups among these have been classified by Miller [6] and Iyer [5]. The most interesting case is undoubtedly $S_{4}$, which is the automorphism group of uncountably many infinite nonabelian groups.

\section{Groups whose central automorphism group is semisimple}

Before stating Theorem 1 we must show how to construct the groups appearing in the classification.

Construction of the groups $G(Q, F, \varepsilon)$. Let $Q$ be a finite group with trivial centre and let $F$ be a torsion-free abelian group. We shall require that $\operatorname{Ext}\left(Q_{a b}, F\right)$ contain an element $\varepsilon$ with the property

$$
C_{\mathrm{Aut} F}(\varepsilon)=1
$$

Here $Q_{a b}=Q / Q^{\prime}$ and Aut $F$ acts on $\operatorname{Ext}\left(Q_{a b}, F\right)$ in the natural way. We shall see in Lemma 1 that a non-trivial $F$ of this type can always be found provided that $Q_{a b}$ is not an elementary abelian 2-group.

Writing $q$ for $\left|Q_{a b}\right|$, we decompose the Schur multiplicator $M(Q)$ into its $q$ and $q^{\prime}-$ components

$$
M(Q)=M(Q)_{q} \oplus M(Q)_{q^{\prime}}
$$

Let $\pi: M(Q) \rightarrow M(Q)_{q^{\prime}}$ be the natural projection. Now put

$$
A=F \oplus M(Q)_{q^{\prime}}
$$

and regard this as a trivial $Q$-module. We deduce from the Universal Coefficients Theorem that

$$
H^{2}(Q, A) \simeq \operatorname{Ext}\left(Q_{a b}, F\right) \oplus \operatorname{Hom}\left(M(Q), M(Q)_{q^{\prime}}\right)
$$

which enables us to identify

$$
\Delta=\varepsilon \oplus \pi
$$

with an element of $H^{2}(Q, A)$. Now choose a central extension $A \longmapsto G \longrightarrow Q$ with cohomology class $\Delta$. Our interest centres on the group

$$
G=G(Q, F, \varepsilon)
$$

Since $Q$ has trivial centre, the centre of $G$ is (the image of) $A$. Thus Inn $G \simeq Q$. Also the torsion-subgroup of the centre is contained in $G^{\prime}$ because $\pi$ is surjective.

Notice that if $Q$ is perfect, $\varepsilon=0$ and $F=1$ : in this case $G=\hat{Q}$, the unique stem cover or full covering group of $Q$. 
Theorem 1. Let $G$ be a group such that Aut $G$ is finite and $\mathrm{Aut}_{c} G$ is semisimple. Then

$$
G \simeq(G(Q, F, \varepsilon) / K) \times D
$$

where $Q, F$ and $\varepsilon$ are as specified in the construction, $K \leqq M(Q)_{q^{\prime}}$ and $D$ is an elementary abelian 2-group whose order is not 4. Furthermore, $F$ is divisible by $\left|M(Q)_{q^{\prime}}: K\right|$, and if $D$ $\neq 1$, then $\left|Q_{a b}\right|$ and $\left|M(Q)_{q^{\prime}}: K\right|$ are odd and $F$ is divisible by 2.

There is a converse to this result.

Theorem 2. Let $G=(G(Q, F, \varepsilon) / K) \times D$ where $Q, F, \varepsilon, K, D$ are as in Theorem 1. Then Aut $_{c} G \simeq$ Aut $D$, which is simple or trivial, and

$$
\text { Aut } G \simeq\left(N_{\text {Aut } Q}(K) \cap \operatorname{St}_{\text {Aut } Q}\left(\varepsilon^{\text {Aut } F}\right)\right) \times \text { Aut } D,
$$

which is finite. Moreover, these isomorphisms arise from the obvious induced mappings.

Here $\varepsilon^{\text {Aut } F}$ denotes the Aut $F$-orbit to which $\varepsilon$ belongs, while $\operatorname{St}_{\text {Aut } Q}\left(\varepsilon^{\text {Aut } F}\right)$ is the stabiliser of that orbit in Aut $Q$.

Proof of Theorem 1. Assume that Aut $G$ is finite and that $\operatorname{Aut}_{c} G \equiv C_{\mathrm{Aut} G}(\operatorname{Inn} G)$ is semisimple. Write $C$ for $Z(G)$, the centre of $G$, and put $Q=G / C \simeq \operatorname{Inn} G$. Then $Q$ is finite and has trivial centre. By a theorem of Nagrebeckii ([7] or [8]) the torsion-subgroup $T$ of $C$ is finite, and we may write

$$
C=F \times T
$$

where $F$ is torsion-free. The theorem will be established in a series of steps.

(a) If $N$ is a characteristic subgroup of $G$ contained in $C$, then $C_{\mathrm{Aut} G}(N) \cap C_{\mathrm{Aut} G}(G / N)=1$ and $\operatorname{Hom}\left((G / N)_{a b}, N\right)=0$.

For $C_{\text {Aut } G}(N) \cap C_{\text {Aut } G}(G / N)$ is normal in Aut $G$ and is contained in Aut $t_{c}$. It is also isomorphic with the abelian group $\operatorname{Hom}\left((G / N)_{a b}, N\right)$ : now apply the semisimplicity of Aut $_{c} G$ to obtain the result.

Take $N$ in (a) to be successively $C$ and $G^{\prime} \cap C=G^{\prime} \cap T$-note that $G^{\prime}$ is finite by Schur's Theorem. We obtain

(b) $\operatorname{Hom}\left(Q_{a b}, T\right)=0=\operatorname{Hom}\left(G_{a b}, G^{\prime} \cap T\right)$. Thus $q=\left|Q_{a b}\right|$ and $|T|$ are relatively prime.

Now let $t=\left|G^{\prime} \cap T\right|$; then $t$ and $q$ are relatively prime. This fact together with the obvious exact sequence $F \longmapsto(G / T)_{a b} \longrightarrow Q_{a b}$ indicates that $F / F^{t}$ is an image of $(G / T)_{a b}$ and hence of $G_{a b}$. Using (b) we derive the following statement.

(c) If $t$ denotes $\left|G^{\prime} \cap T\right|$, then $F=F^{t}$.

The exact sequence $T / G^{\prime} \cap T \longrightarrow(G / F)_{a b} \longrightarrow Q_{a b}$ shows that $T / G^{\prime} \cap T$ is an image of $G_{a b}$. Consequently $\operatorname{Hom}\left(T / G^{\prime} \cap T, G^{\prime} \cap T\right)=0$ and we can state

(d) $\left|G^{\prime} \cap T\right|$ and $\left|T: G^{\prime} \cap T\right|$ are relatively prime. 
It is therefore possible to write $T=\left(G^{\prime} \cap T\right) \times D$ for some $D$. This may be used to produce a direct decomposition of $G$.

(e) $G=G_{1} \times D$ where $D$ is a finite abelian group; the torsion-subgroup of $Z\left(G_{1}\right)$ equals $G^{\prime} \cap T=G_{1}^{\prime} \cap T$.

Observe that $G^{\prime} C=\left(G^{\prime} F\right) \times D$ and $\left|G: G^{\prime} C\right|=\left|Q_{a b}\right|$ is relatively prime to $|D|$. This is wellknown to imply that $G$ splits over $D$, so we can write $G=G_{1} \times D$. Since $C=Z(G)$ $=Z\left(G_{1}\right) \times D$, we have $T=T_{1} \times D$ where $T_{1}$ is the torsion-subgroup of $Z\left(G_{1}\right)$. Now $G^{\prime}$ $=G_{1}^{\prime}$; hence $G^{\prime} \cap T \leqq T_{1}$. Finally $T_{1}=T_{1} \cap\left(\left(G^{\prime} \cap T\right) \times D\right)=G^{\prime} \cap T=G_{1}^{\prime} \cap T$.

(f) The subgroups $G_{1}$ and $D$ are characteristic in $G$. Thus Aut $G \simeq$ Aut $G_{1} \times$ Aut $D$ and Aut $_{c} G=$ Aut $_{c} G_{1} \times$ Aut $D$.

In the first place $D$ is characteristic in $T$, and hence in $G$, since $|D|$ and $|T: D|$ are relatively prime. Applying (a) with $N=D$, we conclude that $\operatorname{Hom}\left(\left(G_{1}\right)_{a b}, D\right)=0$, which implies that $G_{1}$ is characteristic in $G$.

(g) $D$ is an elementary abelian 2-group whose order is not 4. If $D \neq 1$, then $q=\left|Q_{a b}\right|$ and $\left|T_{1}\right|$ are odd, and $F=F^{2}$.

We see from (f) that Aut $D$ is semisimple. Since the automorphism $x \mapsto x^{-1}$ belongs to the centre of Aut $D$, it must be trivial and $D$ is an elementary abelian 2-group; clearly $|D| \neq 4$. Now suppose that $D \neq 1$. Then $q$ is odd because $(q,|T|)=1$. Also $\left|T_{1}\right|$ is odd since $\left(\left|T_{1}\right|,|D|\right)=1$. Finally, it is easy to see that $F / F^{2}$ is an image of $G / D$; since $\operatorname{Hom}\left((G / D)_{a b}, D\right)=0$, it follows that $F=F^{2}$.

Because of (e) and (f) we shall assume as a matter of convenience that $D=1$ and $G$ $=G_{1}$, so $T=G^{\prime} \cap C$.

Let $\Delta$ denote the cohomology class of the central extension $C \succ G \longrightarrow Q$. Now there is a splitting

$$
H^{2}(Q, C) \simeq \operatorname{Ext}\left(Q_{a b}, F\right) \oplus \operatorname{Hom}(M(Q), T)
$$

which is natural in this case because $\left(\left|Q_{a b}\right|,|T|\right)=1$. We can therefore make the identification

$$
\Delta=\varepsilon \oplus \delta
$$

where $\varepsilon \in \operatorname{Ext}\left(Q_{a b}, F\right)$ and $\delta \in \operatorname{Hom}(M(Q), T)$. Notice that $\delta$ is surjective since $\operatorname{Im} \delta$ $=G^{\prime} \cap C=T$.

If $\gamma \in$ Aut $C$ and $\kappa \in$ Aut $Q$, a necessary and sufficient condition for there to be an automorphism of $G$ which induces $\gamma$ and $\kappa$ is that

$$
\Delta \gamma_{*}=\Delta \kappa^{*}
$$

(see [9], Lemma 2.2). Now $T$ is characteristic in $C$, as is $F$ since it is divisible by $|T|$. Therefore $\gamma$ may be represented by a pair $(\varphi, \tau)$ where $\varphi \in$ Aut $F$ and $\tau \in$ Aut $T$. Then (1) is equivalent to the two conditions

$$
\varepsilon \varphi_{*}=\varepsilon \kappa^{*} \text { and } \delta \tau=\delta \kappa^{*}
$$


The conditions for $\gamma=(\varphi, \tau)$ to be induced by a central automorphism of $G$ are obtained by setting $\kappa=1$ in (2). But $\delta \tau=\delta$ implies that $\tau=1$ because $\delta$ is surjective. Therefore these conditions become $\tau=1$ and ' $\epsilon C_{\mathrm{Aut} F}(\varepsilon)$. Consequently the assignment $\alpha \mapsto \varphi$ determines an epimorphism from $\mathrm{Aut}_{c} G$ to $C_{\mathrm{Aut} F}(\varepsilon)$; indeed this is an isomorphism since $\operatorname{Hom}\left(Q_{a b}, C\right)=0$.

It follows that $C_{\mathrm{Aut} F}(\varepsilon)$ is a finite semisimple group. However, by Lemma 6 of [10] this group has exponent dividing 12 , so it is soluble. Therefore $C_{\mathrm{AutF}}(\varepsilon)$ must be trivial and we have established

(h) Aut $_{\mathrm{c}} G \simeq C_{\mathrm{Aut} F}(\varepsilon)=1$.

It is now possible to identify the group $G$.

(i) $G \simeq G(Q, F, \varepsilon) / K$ where $K=(\operatorname{Ker} \delta) \cap M(Q)_{q^{\prime}}$.

Let $\delta$ denote the restriction of $\delta: M(Q) \rightarrow T$ to $M(Q)_{q^{\prime}}$. This is still surjective because $M(Q)_{q} \leqq \operatorname{Ker} \delta$ by (b). Now use $1 \oplus \delta: A=F \oplus M(Q)_{q^{\prime}} \rightarrow C$ to form the push-out diagram

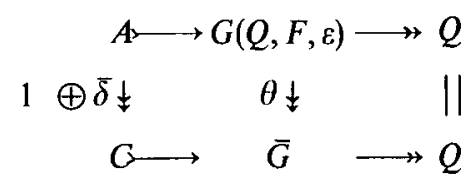

The cohomology class of the lower extension is

$$
(\varepsilon \oplus \pi)(1 \oplus \delta)_{*}=\varepsilon \oplus \pi \bar{\delta}=\varepsilon \oplus \delta=\Delta .
$$

(Recall that $\pi$ is the natural projection from $M(Q)$ to $M(Q)_{q}$ ). Consequently the lower extension is equivalent to $C \longmapsto G \longrightarrow Q$. Since $\operatorname{Ker} \theta=(\operatorname{Ker} \delta) \cap M(Q)_{q^{\prime}}=K$, we have $G \simeq G(Q, F, \varepsilon) / K$ and $T \simeq M(Q)_{q^{\prime}} / K$.

Proof of Theorem 2. Let $G_{1}$ denote the group $G(Q, F, \varepsilon) / K$; then $G=G_{1} \times D$. Also $C$ $=Z(G)=(A / K) \times D$ since $Z(Q)=1$. The hypotheses on $Q_{a b}, F$ and $D$ imply that $\operatorname{Hom}\left(G_{1}, D\right)=0=\operatorname{Hom}\left(D, Z\left(G_{1}\right)\right)$, from which it follows that $G_{1}$ and $D$ are characteristic in $G$. Therefore Aut $G \simeq$ Aut $G_{1} \times$ Aut $D$ and Aut $_{c} G \simeq \operatorname{Aut}_{c} G_{1} \times$ Aut $D$, both isomorphisms arising from the natural induced mappings. Henceforth we shall assume that $D=1$ and $G_{1}=G$.

As in step $(h)$ of the preceding proof it may be shown that $\mathrm{Aut}_{c} G \simeq C_{\text {Aut } F}(\varepsilon)$. Therefore Aut $_{c} G=1$ and the canonical homomorphism Aut $G \rightarrow$ Aut $Q$ is injective. It remains to determine the image.

Consider the conditions (2) for $\gamma=(\varphi, \tau) \in$ Aut $C$ and $\kappa \in$ Aut $Q$ to be induced by an automorphism of $G$; here of course $\varphi \in$ Aut $F, \tau \in$ Aut $T$ and $T=M(Q)_{q} / K$. The condition $\varepsilon \varphi_{*}=\varepsilon \kappa^{*}$ is equivalent to $\kappa$ being in $\mathrm{St}_{\mathrm{Aut} Q}\left(\varepsilon^{\mathrm{Aut} F}\right)$. The second condition $\delta \tau=\delta \kappa^{*}=\kappa_{*} \delta$ implies that $\kappa$ normalises $\operatorname{Ker} \delta$. In this instance $\delta$ is the composite of the projection $\pi: M(Q) \rightarrow M(Q)_{q^{*}}$ and the canonical homomorphism $M(Q)_{q^{\prime}} \rightarrow M(Q)_{q^{\prime}} / K$; thus $\operatorname{Ker} \delta$ $=M(Q)_{q} \oplus K$ and $\kappa$ normalises $K$. Conversely, if $\kappa \in N_{\text {Aul } Q}(K)$, then $\kappa$ normalises $\operatorname{Ker} \delta$ and, since $\delta$ is surjective, we may define $\tau \in$ Aut $T$ by the rule $(x \delta)^{\tau}=\left(x \kappa_{*}\right) \delta$; this is welldefined by choice of $\kappa$. 
The preceding considerations demonstrate that $\kappa \in$ Aut $Q$ lifts to $G$ precisely when $\kappa$ belongs to $N_{\text {Aut } Q}(K) \cap \mathrm{St}_{\mathrm{Aut} Q}\left(\varepsilon^{\mathrm{Aut} F}\right)$; this, then, is the image sought. The proof of the theorem is now complete.

Lemma 1. Let $Q$ be a finite group with trivial centre. Then there exists an infinite group $G(Q, F, \varepsilon)$ if and only if $Q_{a b}$ is not an elementary abelian 2-group.

Proof. In the first place, should $Q_{a b}$ be an elementary abelian 2-group, the automorphism $x \mapsto x^{-1}$ of $F$ will operate trivially on $\operatorname{Ext}\left(Q_{a b}, F\right)$. The condition $C_{\mathrm{Aut} F}(\varepsilon)$ $=1$ must then force $F$ to be trivial. Conversely assume that $Q_{a b}$ has exponent $e>2$. It was shown in [9] (proof of Theorem 6.1) that there is a torsion-free abelian group $F$ with an element $f$ such that $C_{\text {Aut } F}\left(f F^{e}\right)=1$. Writing $Q_{a b} \simeq \underset{i=1,2, \ldots, k}{\operatorname{Dr}} \mathbb{Z}_{e_{i}}$ where $e_{i}$
divides $e_{i+1}$ and $e_{k}=e$, we have

$$
\operatorname{Ext}\left(Q_{a b}, F\right) \simeq \underset{i=1,2, \ldots, k}{\operatorname{Dr}}\left(F / F^{e_{i}}\right)
$$

Denote by $\varepsilon$ the element of $\operatorname{Ext}\left(Q_{a b}, F\right)$ that corresponds to $\left(f F^{e_{1}}, f F^{e_{2}}, \ldots, f F^{e_{k}}\right)$. Then $C_{\text {Aut } F}(\varepsilon)=C_{\text {Aut } F}\left(f F^{e}\right)=1$. We may therefore employ this $F$ and $\varepsilon$ to construct an infinite group $G(Q, F, \varepsilon)$.

\section{Finite semisimple groups as automorphism groups}

By combining Theorems 1 and 2 we are able to give a criterion for a finite semisimple group $H$ to be an automorphism group: there must exist $Q \triangleleft H, K \leqq M(Q)$, $F, \varepsilon \in \operatorname{Ext}\left(Q_{a b}, F\right)$ and $D$ as in Theorem 1 such that $H$ is isomorphic with $\left(\mathrm{St}_{\text {Aut } Q}\left(\varepsilon^{\text {Aut } F}\right) \cap N_{\text {Aut } Q}(K)\right) \times$ Aut $D$.

If there are no normal subgroups whose abelianisation has exponent 3 or more, then by Lemma 1 the group $F$ must be 1 and we are left with the purely internal condition $H \simeq N_{\text {Aut } Q}(K) \times$ Aut $D$.

Here is an illustration of how these techniques may be used in an explicit case.

Lemma 2. Let $S$ be a finite (non-abelian) simple group with cyclic multiplicator. Then there is a group $G$ such that Aut $G \simeq S \backsim \mathbb{Z}_{2}$ if and only if $S$ is complete. Moreover in this case $G \simeq G_{1} \times D$ where $|D| \leqq 2$ and either (i) $G_{1} \simeq \overline{S \times S} / K$ where $K$ is a subgroup of $M(S) \oplus M(S)$ invariant under the involution $(a, b) \mapsto(b, a)$ and $|M(S) \oplus M(S): K|$ is odd if $D$ $\neq 1$, or (ii) $G_{1} \simeq \widehat{S \cup \mathbb{Z}}_{2} / K$ where $\left|M\left(S \backsim \mathbb{Z}_{2}\right): K\right|$ is odd.

Proof. Let $G$ be a group satisfying Aut $G \simeq S \backsim \mathbb{Z}_{2}$; then it is clear that $G$ cannot be abelian. Put $C=Z(G)$ and $Q=G / C$. There are two possibilities to consider: $Q \simeq S \times S$ and $Q \simeq S \backsim \mathbb{Z}_{2}$.

Suppose first that $Q \simeq S \times S$. Then Aut $Q \simeq($ Aut $S) \backsim \mathbb{Z}_{2}, \quad Q_{a b}=1$ and $M(Q) \simeq M(S) \oplus M(S)$. By Theorems 1 and 2 we have $G \simeq(\hat{Q} / K) \times D$ where $|D| \leqq 2$ and $|M(Q): K|$ is odd if $D \neq 1$. Also Aut $G \simeq N_{\text {Aut } Q}(K)$. Let $\alpha \in$ Aut $S$; then $(\alpha, \alpha) \in$ Aut $Q$ maps each element of $M(Q)$ to a power since $M(S)$ is cyclic. Hence $(\alpha, \alpha) \in N_{\text {Aut } Q}(K) \simeq S \backsim \mathbb{Z}_{2}$, 
from which it follows that $\alpha \in \operatorname{Inn} S$. Hence $S$ is complete and $N_{\text {Aut } Q}(K)=$ Aut $Q \simeq S \mho \mathbb{Z}_{2}$. Thus $K$ is left invariant by $(a, b) \mapsto(b, a)$.

Now assume that $Q \simeq S \backsim \mathbb{Z}_{2}$. Then $\left|Q_{a b}\right|=2$ and in fact $M(Q) \simeq M(S)$. Applying Theorem 1 again and Lemma 1 , we obtain Aut $G \simeq(\hat{Q} / K) \times D$ where $|D| \leqq 2$ and $|M(Q): K|$ is odd. Also Aut $G \simeq N_{\text {Aut } Q}(K)=$ Aut $Q \simeq($ Aut $S) \backsim \mathbb{Z}_{2}$. So once again $S$ is complete.

Conversely Theorem 2 shows that in both cases Aut $G \simeq S \backsim \mathbb{Z}_{2}$.

\section{Completely reducible automorphism groups}

Theorem 1 assumes a much simpler form if the group Aut $G$ is completely reducible.

Theorem 3. Let $H$ be a finite completely reducible group. Then there is a group $G$ satisfying Aut $G \simeq H$ if and only if there are direct decompositions $H \simeq Q \times P S L(r, 2)$ and $G \simeq(\hat{Q} / K) \times D$ where $Q$ is a finite completely reducible group, $K$ is a subgroup of $M(Q)$ with the property $N_{\mathrm{Out} Q}(K)=1$ and $D$ is an elementary abelian 2-group of order $2^{r} \neq 4$, with the additional stipulation that $|M(Q): K|$ be odd if $D \neq 1$.

Proof. Assume that Aut $G \simeq H$ and write $Q=G / Z(G)$. Then $Q$ is also completely reducible and $Q_{a b}=1$. Theorem 1 shows that $G \simeq(\hat{Q} / K) \times D$ and Theorem 2 that Aut $G \simeq N_{\text {Aut } Q}(K) \times$ Aut $D$ : here $K \leqq M(Q), D$ is elementary abelian of order $2^{r} \neq 4$ and $|M(Q): K|$ is odd if $D \neq 1$. Now $C_{\text {Aut } Q}(\operatorname{Inn} Q)=1$ since $Z(Q)=1$. However Inn $Q$ is a direct factor of the group $N_{\text {Aut } Q}(K)$ by a well-known property of completely reducible groups. Hence $N_{\text {Aut } Q}(K)=\operatorname{Inn} Q$ and $N_{\text {Out } Q}(K)=1$.

The converse follows from Theorem 2 since Inn $Q \simeq Q$.

Theorem 3 takes a particularly satisfying form for simple groups.

Theorem 4. Let $S$ be a finite (non-abelian) simple group. Then there is a group $G$ satisfying Aut $G \simeq S$ if and only if either

(a) $G$ is an elementary abelian 2-group of order $2^{r}>4$ and $S \simeq P S L(r, 2)$

or

(b) there is a subgroup $K$ of $M(S)$ such that $N_{\text {Out } S}(K)=1$ and $G \simeq \hat{S} / K$ or $(\hat{S} / K) \times \mathbb{Z}_{2}$ where in the second case $|M(S): K|$ is odd.

The question arises: for which finite simple groups $S$ does $M(S)$ have a subgroup $K$ such that

$$
N_{\text {Out } S}(K)=1 ?
$$

Of course, if $S$ is complete, this is automatic for any $K$. However, it seems to be difficult to fulfil (3) if $S$ is incomplete. Certainly $M(S)$ cannot be cyclic in this case, which eliminates the sporadic groups, the alternating groups and the groups of Lie type with 
the following exceptions:

$$
A_{2}(4), D_{4}(2),{ }^{2} A_{5}(2),{ }^{2} E_{6}(2),{ }^{2} A_{3}(3),{ }^{2} B_{2}(8)
$$

and

$$
D_{2 n}(q),(q \text { odd }, n \geqq 2) \text {. }
$$

(For the multiplicators of the known finite simple groups see [3]).

However, a detailed check* of these groups reveals only one which has the property in question, the Suzuki group

$$
{ }^{2} B_{2}(8) \equiv S z(8)
$$

If we denote this group by $S$, then

$$
M(S) \simeq \mathbb{Z}_{2} \oplus \mathbb{Z}_{2} \quad \text { and } \quad \text { Out } S \simeq \mathbb{Z}_{3}
$$

here Out $S$ permutes the three subgroups of $M(S)$ that have order 2 . This information can easily be read off from results of Alperin and Gorenstein [1]. Hence there are three candidates for $K$, but it is not difficult to show that they give rise to isomorphic groups.

Corollary. Let $S$ be a finite simple group of known type. Then there is a group $G$ satisfying Aut $G \simeq S$ if and only if one of the following is true:

(a) $S \simeq P S L(r, 2), r>2$, and $G$ is an elementary abelian 2-group of order $2^{r}$.

(b) $S$ is complete and $G \simeq \hat{S} / K$ or $(\hat{S} / K) \times \mathbb{Z}_{2}$ where $K$ is any subgroup of $M(S)$ and in the second case $|M(S): K|$ is odd.

(c) $S \simeq S z(8)$ and $G \simeq \hat{S} / K$ where $K$ is a subgroup of $M(S)$ with order 2 .

\section{Covering groups of simple groups}

If $S$ is a finite simple group and $K$ is a proper subgroup of $M(S)$, then $\hat{S} / K$ will be called a non-trivial covering group of $S$.

Theorem 5. If $S$ is a finite simple group of known type, no non-trivial covering group of $S$ can be an automorphism group.

Proof. Suppose that $G$ is a group satisfying Aut $G \simeq \hat{S} / K$ where $K<M(S)=Z(\hat{S})$. It is well-known that $\hat{S}$ is perfect ([11]), so Aut $G$ is perfect. From this it follows that every proper normal subgroup of $\hat{S} / K$ is contained in $Z(\hat{S} / K)=M(S) / K$. Hence either Inn $G$ $=$ Aut $G$ or Inn $G \leqq Z$ (Aut $G$ ). Let $C$ denote the centre of $G$.

If Inn $G=$ Aut $G$, then $G / C$ is perfect. Consequently $G / C$ has trivial centre and $M(S)$ $=K$, a contradiction. It follows that $\operatorname{Inn} G \leqq Z$ (Aut $G$ ) and Aut $G=$ Aut $_{c} G$. Clearly $G$ is nilpotent of class at most 2 .

*Here I am much indebted to R. L. Griess. 
In what follows it is necessary to know that the multiplicators of the known finite simple groups are cyclic with certain exceptions in which for either $p=2$ or $p=3$ the $p$ components have rank 2 and the $p^{\prime}$-components are cyclic.

Consider first the case where $G$ is abelian. Write $G=F \times T$ where $F$ is torsion-free and $T$ is finite. Then Aut $F$ is finite and hence soluble by a theorem of Hallett and Hirsch [4]. Since Aut $F$ is an image of Aut $G$, we deduce that Aut $F=1$ and $F=1$, so that $G$ is finite. Evidently we may assume that $G$ is a $p$-group.

If $G$ is elementary, then Aut $G \simeq G L(n, p)$, which is perfect and non-trivial only if $p=2$ and $n>2$, when it is simple. But this would force $K$ to equal $M(S)$. Hence $G$ is not elementary and there is a decompositon $G=A \times B$ in which $B$ is homocyclic of exponent $p^{e}>p$ while $A^{p^{e-1}}=1$. Now $\operatorname{Hom}\left(G / G^{p}, G^{p}\right)$ is isomorphic with a normal abelian subgroup of Aut $G$ and hence with a factor of $M(S)$. Therefore the product of the ranks of $G / G^{p}$ and $G^{p}$ is at most 2 , which shows that $A$ and $B$ are cyclic. But it is easy to see that Aut $G$ cannot be perfect in this case.

Now suppose that $G$ is not abelian. Then $G / C$ is not cyclic and so $M(S)$ cannot be cyclic. Hence for $p=2$ or 3 the $p$-component of $M(S)$ has rank 2 and the $p^{\prime}$-component is cyclic. The group $\operatorname{Hom}(G / C, C)$ is isomorphic with a normal abelian subgroup of Aut $G$ and hence with a factor of $M(S)$. This means that the $p^{\prime}$-component of $G / C$ is cyclic and hence trivial, so that $G / C$ is a non-cyclic $p$-group. In addition, if $T$ is the torsion-subgroup of $C$, then $T_{p}$ is cyclic.

Let $q$ be a prime other than $p$. It is easy to see that $T_{q}$ is a direct factor of $G$, say $G$ $=T_{q} \times U$. Suppose that $T_{q}$ is not cyclic. Now Hom $\left(\left(G / T_{q}\right)_{a b}, T_{q}\right)$ is isomorphic with a normal abelian $q$-subgroup of Aut $G$ and so of $M(S) / K$. Since $M(S)_{q}$ is cyclic, $\left(G / T_{q}\right)_{a b}$ must be divisible by $q$. This implies that $U$ is characteristic in $G$ and Aut $G$ $=$ Aut $T_{q} \times$ Aut $U$. However $\widehat{S} / K$ is obviously indecomposable. Therefore $|U| \leqq 2$ and $G$ is abelian.

The preceding argument permits us to assume that $T$ is cyclic. Because Aut $G$ is perfect it will then operate trivially on $T$.

Next $G$ is nilpotent and Aut $G$ is finite. By Corollary 5.4 of [9] it follows that Aut $(C / T)$ is finite; therefore Aut $(C / T)$ is soluble. But Aut $G$ is perfect, so it must operate trivially on $C / T$, as well as on $T$. Since Aut $G=$ Aut $_{c} G$, we deduce that Aut $G$ is nilpotent, which gives the contradiction Aut $G=1$.

\section{Alternating and symmetric groups}

The alternating group $A_{n}$ is very rarely an automorphism group.

Theorem 6. There is a group $G$ satisfying Aut $G \simeq A_{n}$ if and only if either $n \leqq 2$ and $|G| \leqq 2$ or $n=8$ and $G$ is elementary abelian group of order 16 .

Proof. Assume that Aut $G \simeq A_{n}$. When $n \leqq 3$, the situation is clear; let $n>3$. If $G$ is abelian, it will have to be an elementary abelian 2-group, of order $2^{r}$ say, since $A_{n}$ has trivial centre; then $P S L(r, 2) \simeq A_{n}$, which occurs only if $r=4$ and $n=8$ ([2]).

Now assume that $G$ is not abelian. Since $A_{n}$ is nev : complete, it follows from the Corollary that $n=4$. Let $C=Z(G)$ and $Q=G / C$. If $C$ is a 4-group, $2 H^{2}(Q, C)=0$ and 
inversion in $C$ extends to a central automorphism of $G$ ([9], Lemma 2.2). But Aut ${ }_{c} G$ $=\operatorname{Inn} G$ in this case. Hence $C$ is an elementary abelian 2-group. Examination of the order of $\operatorname{Hom}(Q, C)$ shows that $|C|=2$ and $|G|=8$, which is impossible.

Finally, if $Q \simeq A_{4}$, it is easy to verify with the aid of Lemma 2.2 of [9] that an outer automorphism of $Q$ can be paired with inversion in $C$ to produce an outer automorphism of $G$, again a contradiction.

For the symmetric group $S_{n}$ the situation is considerably more complicated. To describe the infinite groups whose automorphism group is $S_{4}$ we shall specialise the construction of Section 2.

Let $Q=A_{4}$; then $Q_{a b} \simeq \mathbb{Z}_{3}$ and $M(Q) \simeq \mathbb{Z}_{2}$. If $F$ is a torsion-free abelian group, $\operatorname{Ext}\left(Q_{a b}, F\right) \simeq F / F^{3}$. Suppose that $F / F^{3}$ contains an element $\varepsilon$ such that

$$
C_{\mathrm{Aut} F}(\varepsilon)=1
$$

Then we shall write with a slight abuse of notation

$$
G(F, \varepsilon)=G(Q, F, \varepsilon)
$$

There are in fact uncountably many non-isomorphic groups $G(F, \varepsilon)([9]$, Theorem 6.1).

Theorem 7. There is a group $G$ with the property Aut $G \simeq S_{n}$ if and only if one of the following is true.

(i) $n=1$ and $|G| \leqq 2$.

(ii) $n=2$ and $G \simeq \mathbb{Z}_{3}, \mathbb{Z}_{4}, \mathbb{Z}_{6}$ or a torsion-free abelian group whose only non-trivial automorphism is $x \mapsto x^{-1}$.

(iii) $n=3$ and $G \simeq \mathbb{Z}_{2} \oplus \mathbb{Z}_{2}$ or $S_{3}$.

(iv) $n=4, G$ is finite and $G \simeq A_{4}, A_{4} \times \mathbb{Z}_{2}, S_{4}, Q_{8}$ or $\hat{A}_{4}=S L(2,3)$.

(v) $n=4, G$ is infinite and $G \simeq G(F, \varepsilon), G(F, \varepsilon) / \mathbb{Z}_{2}$ or $\left(G(F, \varepsilon) / \mathbb{Z}_{2}\right) \times \mathbb{Z}_{2}$ where in the first and third cases $F=F^{2}$.

(vi) $n \geqq 5, n \neq 6$ or 7 and $G \simeq A_{n}, A_{n} \times \mathbb{Z}_{2}, S_{n}$ or $\hat{A}_{n}$.

(vii) $n=7$ and $G \simeq A_{7}, A_{7} \times \mathbb{Z}_{2}, S_{7}, \hat{A}_{7}, \hat{A}_{7} / \mathbb{Z}_{2}, \hat{A}_{7} / \mathbb{Z}_{3}$ or $\left(\hat{A}_{7} / \mathbb{Z}_{2}\right) \times \mathbb{Z}_{2}$.

Notice the corollaries: $S_{6}$ is not an automorphism group; $S_{2}$ and $S_{4}$ are the only finite symmetric groups that are the automorphism groups of infinite groups.

Proof of Theorem 7. The first two statements are elementary and we omit the proof. Assume from now on that $n>2$. If $G$ is abelian, it is an elementary abelian 2-group, of order $2^{r}$ say, and $S_{n} \simeq P S L(r, 2)$, which can only happen if $r=2$ and $n=3$. Assume henceforth that $G$ is not abelian. Writing $C$ for $Z(G)$ and $Q$ for $G / C$, we have $Q \simeq A_{n}$ or $S_{n}$ with the additional possibility that $Q$ is a 4 -group if $n=4$.

Suppose that $Q \simeq S_{n}$, so that $\operatorname{Inn} G=\operatorname{Aut} G$ and Aut $_{c} G=1$. Thus Theorem 1 applies. Since $\left|Q_{a b}\right|=2$, the group $G$ is finite. Also $M(Q)=0$ if $n=3$ and $M(Q) \simeq \mathbb{Z}_{2}$ otherwise. It follows that $G \simeq Q$ and $S_{n} \simeq$ Aut $G \simeq$ Aut $Q$. This tells us that $n \neq 6$. It also disposes of the case $n=3$. Assume therefore that $n \geqq 4$ and $Q \nVdash S_{n}$. 
Now let $n=4$. If $Q$ is a 4-group, then, as in the previous proof, $|G|=8$, which can only mean that $G \simeq Q_{8}$. If $Q \simeq A_{4}$, then $G \simeq G(F, \varepsilon), G(F, \varepsilon) / \mathbb{Z}_{2}$ or $\left(G(F, \varepsilon) / \mathbb{Z}_{2}\right) \times \mathbb{Z}_{2}$ by Theorem 1: notice that $\mathrm{St}_{\text {Au } Q}\left(\varepsilon^{\text {AuI } F}\right)=$ Aut $Q$ in this case. When $F=1$, we obtain the finite groups $\hat{A}_{4}, A_{4}, A_{4} \times \mathbb{Z}_{2}$.

If $n \geqq 5$, then $Q \simeq A_{n}$ and $Q_{a b}$ is trivial. Hence $G$ is finite. Also $|M(Q)|=2$, unless $n=6$ or 7 when $|M(Q)|=6$. By Theorem 1 we have $G \simeq(\hat{Q} / K) \times D$ where $|D| \leqq 2$. Allowing for the various subgroups of $M(Q)$, we obtain the result.

\section{REFERENCES}

1. J. L. Alperin and D. Gorenstein, The multiplicators of certain simple groups, Proc. Amer. Math. Soc. 17 (1966), 515-519.

2. E. Artin, The orders of the classical simple groups, Comm. Pure Appl. Math. 8 (1955), 455472.

3. R. L. Griess, Schur multipliers of the known finite simple groups I, II, Bull. Amer. Math. Soc. 78 (1972), 68-71, Proc. Symposia Pure Math. 37 (1980), 279-282.

4. J. T. HaLleTt and K. A. HirsCh, Torsion-free groups having finite automorphism groups I, J. Algebra 2 (1965), 287-298.

5. H. IYeR, On solving the equation Aut $(X)=G$, Rocky Mountain J. Math. 9 (1979), 653-670.

6. G. A. Miller, On the groups which have the same group of isomorphisms, Trans. Amer. Math. Soc. 1 (1900), 395-401.

7. V. T. NaGRebeckil, On groups with a finite number of automorphisms, Mat. Sb. 86 (1971), $571-577=$ Math. USSR-Sb 15 (1971), 568-575.

8. V. T. NAGREBECKII, On the periodic part of a group with a finite number of automorphisms, Dokl. Akad. Nauk SSSR 205 (1972), 519-521 = Soviet Math. Dokl. 13 (1972), 953-956.

9. D. J. S. Robrnson, A contribution to the theory of groups with finitely many automorphisms, Proc. London Math. Soc. (3) 35 (1977), 34-54.

10. D. J. S. Robinson, Infinite torsion groups as automorphism groups, Quart. J. Math. (2) 30 (1979), 351-364.

11. U. Stammbach, Homology in Group Theory (Lecture Notes in Mathematics Vol. 359, Springer, Berlin, 1973).

Department of Mathematics

UNIVERSITY OF ILLINOIS

URBANA ILLINOIS 61801

U.S.A. 